\title{
Cardiomegaly in a premature neonate after venous umbilical catheterization
}

\author{
Luregn Jan Schlapbach • Jean-Pierre Pfammatter • \\ Mathias Nelle $\cdot$ Felicity Jane McDougall
}

Received: 31 January 2008 / Accepted: 25 February 2008 / Published online: 1 April 2008

(C) Springer-Verlag 2008

\begin{abstract}
Umbilical venous catheters allow rapid central access in neonates, but may be associated with various complications. We present a case of a newborn with pericardial effusion following umbilical venous catheterization. An extremely low birth weight infant was intubated for respiratory distress syndrome and had umbilical venous and arterial lines in place. Massive cardiomegaly was noted on the subsequent chest X-ray. Echocardiography revealed a large pericardial effusion without signs of tamponade. After removing the catheter, the effusion gradually resolved. While pericardial effusion is a well-known complication of percutaneous long central lines, only a few case reports have documented sudden cardiovascular compromise associated with umbilical venous catheters. Pericardial effusion may be asymptomatic and should be suspected in infants with central catheters and progressive cardiomegaly. The prompt removal of catheters and, if signs of cardiac tamponade are present, emergency pericardiocentesis may prove to be life-saving.
\end{abstract}

L. J. Schlapbach $\cdot$ M. Nelle $\cdot$ F. J. McDougall

Division of Neonatology, Department of Pediatrics,

University of Berne,

Berne, Switzerland

\section{J. Schlapbach $(\bowtie)$}

Neonatology and Pediatric Intensive Care,

University Children's Hospital Zürich,

Steinwiesstr. 75,

8032 Zürich, Switzerland

e-mail: luregn.schlapbach@bluewin.ch

J.-P. Pfammatter

Division of Pediatric Cardiology, Department of Pediatrics,

University of Berne,

Berne, Switzerland
Keywords Central venous catheterization · Umbilical vein . Prematurity $\cdot$ Pericardial effusion $\cdot$ Cardiac tamponade
Abbreviations
UVC Umbilical venous catheter
UAC Umbilical arterial catheter
PICC Peripherally inserted central catheter

\section{Introduction}

Umbilical venous catheters (UVC) are frequently used in neonates requiring hyperosmotic parenteral nutrition, catecholamines, or when no peripheral access can be established. They allow rapid central access, but may be associated with various complications [2]. Clinicians are particularly aware of catheter-associated infections and thrombosis. Due to the widespread use of umbilical lines, neonatologists should keep rare complications in mind as well. We present a case of a newborn with pericardial effusion following venous umbilical catheterization.

\section{Case description}

An extremely premature infant weighing $590 \mathrm{~g}$ was born at 25 weeks gestational age by cesarean section for severe maternal pre-eclampsia and deteriorating fetal Doppler studies. The baby was intubated for respiratory distress syndrome within the first hour of life and umbilical venous and arterial lines were placed (UVC 3.5 charrière singlelumen, UAC 2.5 charrière, Argyle ${ }^{\mathrm{TM}}$ polyurethane, Tyco Healthcare, Tullamore, Ireland). Blood could be easily aspirated from both catheters. The position of the catheters, 


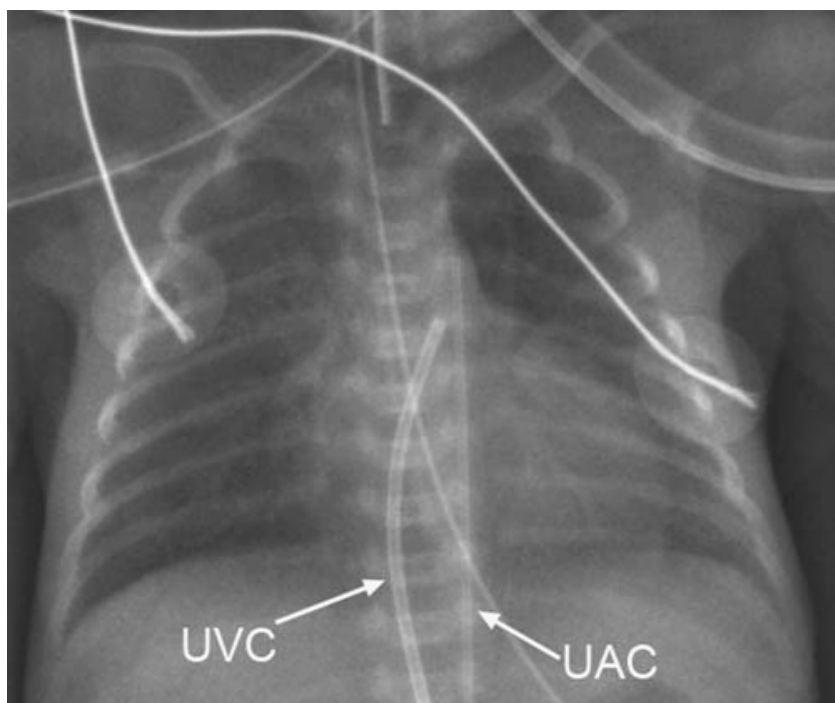

Fig. 1 Chest X-ray on day one after the intubation and insertion of venous (UVC) and arterial (UAC) umbilical lines

both of which had been inserted too far (Fig. 1), was corrected according to the chest X-ray by $1.5 \mathrm{~cm}$ (UVC) and $2 \mathrm{~cm}$ (UAC). After receiving porcine surfactant (Curosurf ${ }^{\circledR}$ ), the infant was successfully weaned. Chest Xray before planned extubation on day three unexpectedly showed cardiomegaly with a lung-to-chest ratio of 0.69 (Fig. 2). Echocardiography was performed immediately and revealed a large echofree pericardial effusion measuring $6 \mathrm{~mm}$ in diameter (Fig. 3). In spite of the effusion, both atrial and ventricular function were adequate, without diastolic indentation of the atrial wall. The UVC tip was floating within the right atrium. On X-ray examination, the UVC was again positioned too high, projecting into the cardiac silhouette (Fig. 2), although its initial position had

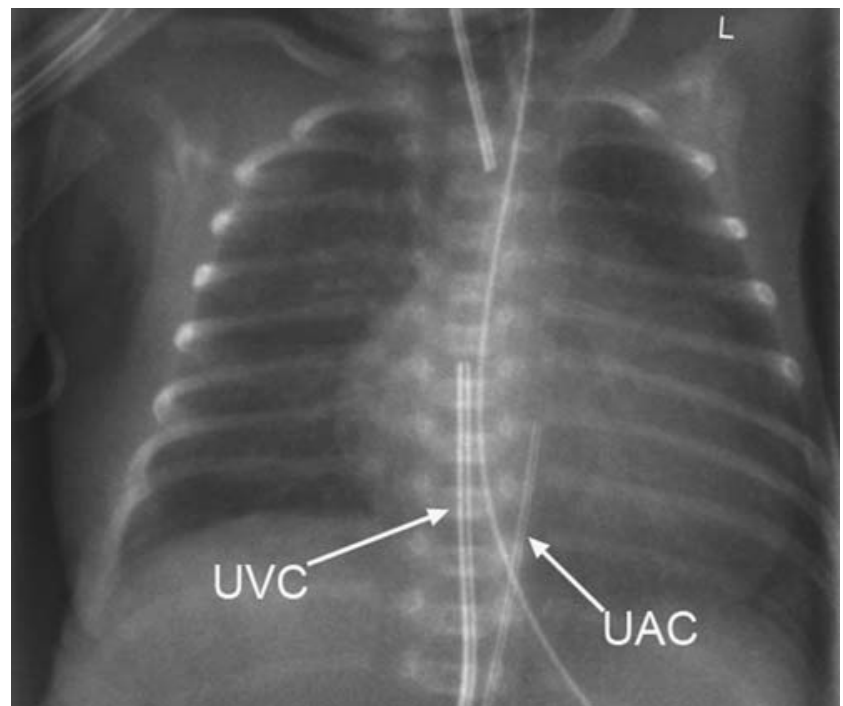

Fig. 2 Incidental finding of cardiomegaly on chest X-ray examination before extubation on day three. $\mathrm{UVC}=$ umbilical venous catheter; $\mathrm{UAC}=$ umbilical arterial catheter

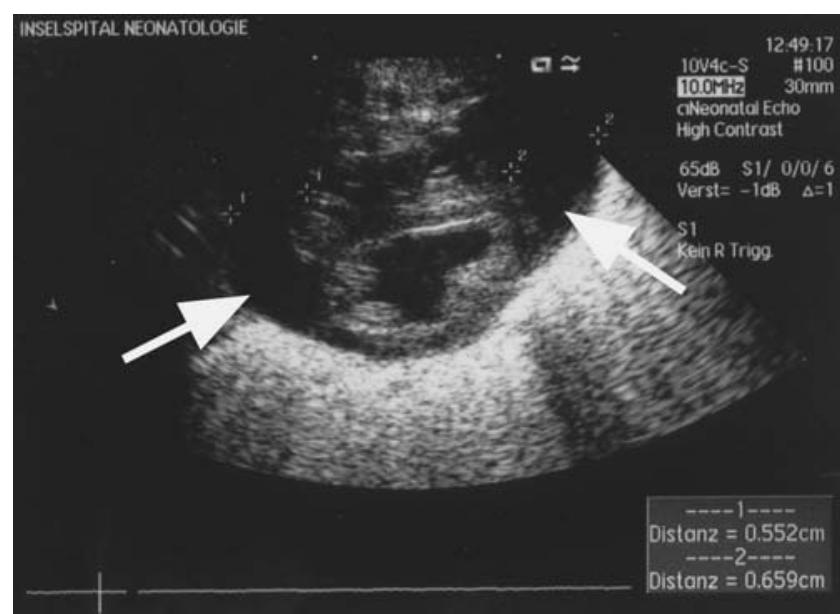

Fig. 3 Echocardiography demonstrating large pericardial effusion (white arrows) on day three

been adequately corrected. Aspiration through the UVC yielded bloody fluid, and blood gas and chemical analysis of the aspirate were consistent with blood and not parenteral nutrition. Ultrasound examination ruled out pleural and abdominal effusions. Stool cultures of the infant and the mother were negative for enterovirus. Maternal serology resulted negative for TORCHS, Parvovirus B19, and Coxiella burneti.

Since the infant remained hemodynamically stable with no signs of cardiovascular compromise, we decided against emergency pericardiocentesis. After removing the UVC, the effusion gradually resolved within a few days, and the infant was successfully extubated. The later clinical course was complicated by bronchopulmonary dysplasia and osteopathy of prematurity. Later cardiac follow-up revealed no functional nor anatomical pathology.

\section{Discussion}

Pericardial effusion is a well-known complication of peripherally inserted central catheters (PICC), with an estimated incidence of 1.8/1,000 catheters [1]. The majority of infants with reported pericardial effusion became acutely symptomatic due to cardiac tamponade and deteriorated rapidly with signs of respiratory distress, cyanosis, tachycardia or bradycardia, mottled skin, and arterial hypotension, finally leading to cardiopulmonary arrest not responsive to standard interventions [1, 3]. Notably, approximately a quarter of the cases were first diagnosed during post-mortem at autopsy [1]. The mortality is very high $(45-65 \%)[1,3]$ and those resuscitated successfully improved only after emergency pericardiocentesis was performed. Analysis of the aspirated liquid usually reflected the composition of the parenteral nutrition. Following a series of case reports on infant deaths attributed to PICC- 
associated cardiac tamponade, guidelines have been published aimed at reducing the risk of cardiac perforation $[3,4,6]$.

Contrary to PICC, the incidence of pericardial effusion associated with UVC is unknown, but case reports have documented sudden cardiovascular compromise in infants with UVC due to pericardial tamponade $[5,8,9]$.

Perforation and catheter migration are thought to occur as a result of both mechanical pressure by the catheter tip repeatedly pushing against the contracting heart wall and endocardial injury caused by hyperosmotic parenteral nutrition fluids $[1,9]$. The transmural diffusion of parenteral nutrition fluids into the pericardial space further contributes to the accumulation of fluid. Contrary to catheter-associated infections and thrombosis, which increase over time, pericardial effusion may occur directly after the insertion of catheters, or later, with a peak at three days following catheter insertion [4]. The malposition of central catheters is considered to be the main risk factor for pericardial effusion, particularly if the catheter tip projects into the right atrium or shows angulation [1, 3, 8]. Umbilical catheters should not be used if the blood does not return freely upon insertion. The catheter tip should be positioned at the junction of the vena cava inferior and right atrium, with the tip lying outside the cardiac silhouette. However, catheter inward migration, as experienced in the present case, has been described and is attributed to retraction of the mummifying cord, changes in abdominal girth, and catheter dislocation during manipulations [7,9]. Therefore, even after correct initial placement, the UVC position should be checked regularly using X-ray or ultrasound.

The differential diagnosis of neonatal pericardial effusion includes immune and nonimmune hydrops fetalis, congenital infections such as TORCHS and Parvovirus B19, and rarely myopericarditis caused by Enteroviridae, mainly Echovirus and Cocksackievirus, or Coxiella burneti.

The present case illustrates that pericardial effusion may progress asymptomatically before hemodynamic changes become evident. The incidence of catheter-associated pericardial effusion may, therefore, be underestimated. Extremely low birth weight infants might be at particular risk due to the thin myocardial wall with relatively large catheters often - as in this case - being initially inserted too far. Considering the potentially lethal complications of UVC, neonatologists should carefully consider the indica- tion for placing UVCs and remove UVCs as soon as possible. Whether percutaneous long lines represent a safer alternative remains unclear, and further prospective studies comparing UVC and PICC are needed [2]. Malpositioned UVCs should be corrected immediately and the position should be verified afterwards. Finally, neonatologists should maintain a high index of suspicion for pericardial tamponade and readily perform echocardiography in acutely ill infants with UVCs.

In conclusion, pericardial effusion may occur asymptomatically after umbilical venous catheterization and should be suspected in infants with central catheters and progressive cardiomegaly. The prompt removal of catheters and, if signs of pericardial tamponade are present, emergency pericardiocentesis may prove to be life-saving.

Conflicts of interest statement None declared.

\section{References}

1. Beardsall K, White DK, Pinto EM, Kelsall AW (2003) Pericardial effusion and cardiac tamponade as complications of neonatal long lines: are they really a problem? Arch Dis Child Fetal Neonatal Ed 88:F292-F295

2. Butler-O'Hara M, Buzzard CJ, Reubens L, McDermott MP, DiGrazio W, D'Angio CT (2006) A randomized trial comparing long-term and short-term use of umbilical venous catheters in premature infants with birth weights of less than 1251 grams. Pediatrics 118:e25-e35

3. Darling JC, Newell SJ, Mohamdee O, Uzun O, Cullinane CJ, Dear PR (2001) Central venous catheter tip in the right atrium: a risk factor for neonatal cardiac tamponade. J Perinatol 21:461-464

4. Nowlen TT, Rosenthal GL, Johnson GL, Tom DJ, Vargo TA (2002) Pericardial effusion and tamponade in infants with central catheters. Pediatrics 110:137-142

5. Onal EE, Saygili A, Koç E, Türkyilmaz C, Okumus N, Atalay Y (2004) Cardiac tamponade in a newborn because of umbilical venous catheterization: is correct position safe? Paediatr Anaesth 14:953-956

6. Pezzati M, Filippi L, Chiti G, Dani C, Rossi S, Bertini G, Rubaltelli FF (2004) Central venous catheters and cardiac tamponade in preterm infants. Intensive Care Med 30:2253-2256

7. Salvadori S, Piva D, Filippone M (2002) Umbilical venous line displacement as a consequence of abdominal girth variation. J Pediatr 141:737

8. Sehgal A, Cook V, Dunn M (2007) Pericardial effusion associated with an appropriately placed umbilical venous catheter. J Perinatol 27:317-319

9. Traen M, Schepens E, Laroche S, van Overmeire B (2005) Cardiac tamponade and pericardial effusion due to venous umbilical catheterization. Acta Paediatr 94:626-628 